\title{
Euler's polyhedron formula - a starting point of today's polytope theory
}

\author{
Günter M. Ziegler and Christian Blatter
}

1. Euler's polyhedron formula, known as

$$
e-k+f=2
$$

(and shown on the commemorative stamp put out by the Swiss Post, Fig. 1), or in more modern notation as

$$
f_{0}-f_{1}+f_{2}=2,
$$

represents the historical starting point of algebraic topology; moreover, it is one of the corner stones of modern $f$-vector theory about which we shall hear more later on. While regular polyhedra, the "Platonic solids", were studied since antiquity, it is Euler's formula that for the first time puts general polyhedra into the center of attention.

In this lecture we start by recounting the story of this formula, but then our attention will gradually shift to $f$-vector theory. The outline of the talk is as follows:

- a precursor (Descartes, 1630),

- the Euler-Poincaré formula for $d$-dimensional polytopes (Schläfli 1852),

- the characterisation of the $f$-vectors of 3-polytopes (Steinitz 1906),

- the characterisation of the $f$-vectors of simplicial $d$-polytopes (Billera \& Lee 1980 and Stanley 1980),

- the characterisation of the $f$-vectors of 4-polytopes (work in progress, still incomplete).

Before we actually begin, a bit of terminology! (Here we are appealing to the threedimensional intuition of the reader.)

By a polytope $P$ we mean the convex hull of a finite set of points in some $\mathbb{R}^{d}$, where it is tacitly assumed that $P$ has nonempty interior. $P$ possesses (proper) faces of dimensions between 0 and $d-1$, where the 0 -dimensional faces (known as the vertices) are the extremal points of $P$, and the $(d-1)$-dimensional faces are called facets. The number of $r$-dimensional faces is generally denoted by $f_{r}(0 \leq r \leq d-1)$. 


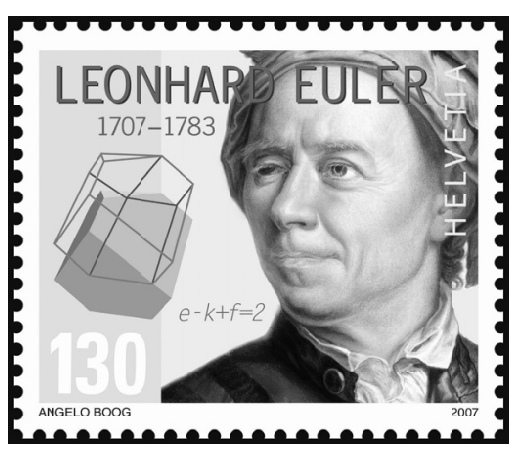

Figure 1

When the vertices are "in general position" no more than $d$ of them lie in the same hyperplane. It follows that in this case all facets and consequently all proper faces of $P$ are simplices of the respective dimensions. Such a polytope is called simplicial. The octahedron in $\mathbb{R}^{3}$ is an example.

There is a second way to obtain a polytope: $P$ may be given as intersection of finitely many closed halfspaces, where it is assumed that this intersection is bounded. It is a basic theorem that this second construction principle leads to the same class of objects as the one described a moment ago. In addition there is a combinatorial duality at work under which, e.g., the octahedron corresponds to the cube. Again: When the defining hyperplanes are "in general position" then no more than $d$ of them will go through the same point. It follows that in this case each vertex lies in exactly $d$ facets. Such a polytope is called simple. Combinatorially, simplicial and simple polytopes are dual to each other.

2. When Descartes died in Stockholm, 11 February 1650, his mathematical "Nachlass" remained in Sweden for a few years, and then was sent to Paris. It arrived there in bad condition but nevertheless was published in part by 1667. In 1676 Leibniz had a chance to look at some of the unpublished material, and among these papers he found a handwritten note "Progymnasmata De Solidorum Elementis" that took his interest, and he copied part of it to take home. Descartes' original note went lost, and Leibniz' copy [2] remained unpublished at the time. It took 200 years until it was found in an uncatalogued part of Leibniz' "Nachlass" at the Royal Library of Hanover. Fig. 2, taken from [4], shows an enlargement from the relevant page. Mathematically it says the following: Consider a polyhedron, i.e., a polytope in $\mathbb{R}^{3}$. Let $\alpha$ be the number of "solid angles" (i.e., vertices), $\phi$ be the number of facets, and $\rho$ be the number of "plane angles" (i.e., angles at the vertices of the facets). By arguing about the sum $\Sigma$ of these plane angles Descartes proves that

$$
\rho=2 \phi+2 \alpha-4
$$

Now each plane angle is formed by two edges, and each edge appears in four plane angles. It follows that $\rho=2 k$, so (3) may be interpreted as a variant of (1) and can indeed be seen as a precursor of Euler's formula. 


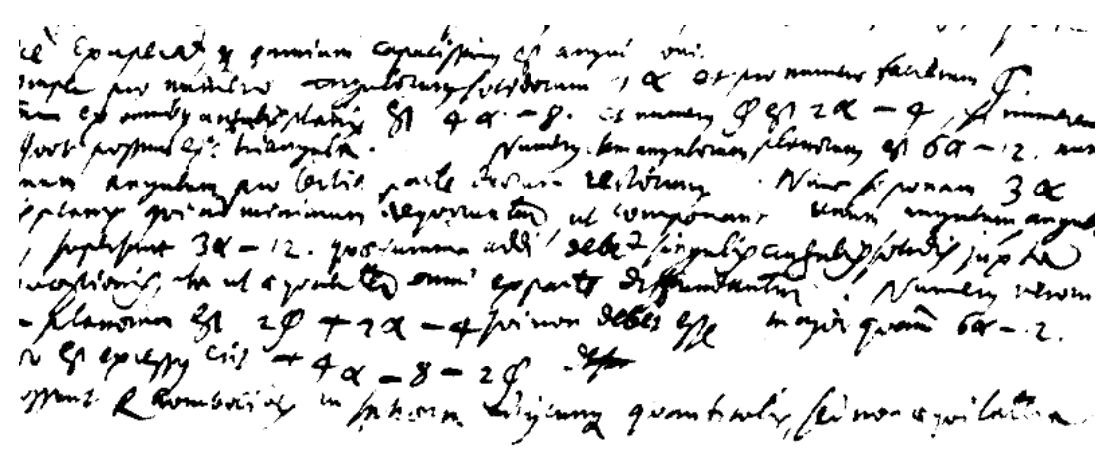

Figure 2

We come to Euler. His formula makes its first appearance in a letter to Goldbach, 14 November 1750 [8]. Maybe in the same year Euler submitted a corresponding note to the Petersburg Academy. It appeared in the Academy's Proceedings of 1752/53 which were published only in 1758. Here is what Euler had to say: Consider any polyhedron, let $S$ be the number of its solid angles, $H$ be the number of its "hedra" (i.e., facets), and $A$ be the number of its "acies" (i.e., edges). Then $S+H=A+2$. Voilà! By the way, Euler seems to be the first to speak about the edges of a polyhedron.

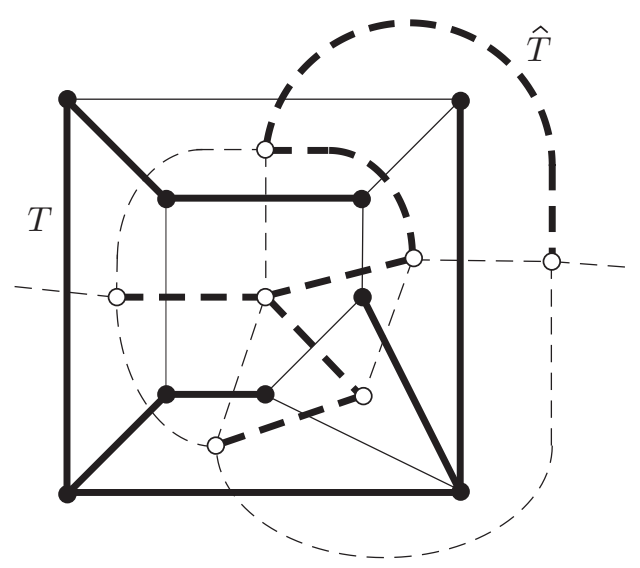

Figure 3

Here is a charming proof of Euler's formula, taken from David Eppstein's “geometry junkyard" [3]: Consider the 1-skeleton $\Gamma$ of $P$ (a planar graph) and in the same figure (Fig. 3) the 1-skeleton $\hat{\Gamma}$ of the "dual" $\hat{P}$ of $P$. Let $T$ be a spanning tree of $\Gamma . T$ does not contain any cycles, so it does not disconnect the plane. Since $T$ is maximal, it follows that there is a tree $\hat{T} \subset \hat{\Gamma}$ such that each edge of $P$ resp. $\hat{P}$ appears either in $T$ or in $\hat{T}$. The vertices of $T$ are the vertices of $P$. So $T$ has $f_{0}$ vertices and $k:=f_{0}-1$ edges. Similarly, the vertices of $\hat{T}$ correspond to the facets of $P$, so $\hat{T}$ has $f_{2}$ vertices and $\hat{k}:=f_{2}-1$ edges. Since $k+\hat{k}=f_{1}$, formula (2) follows. 
One of the pioneering figures in the higher-dimensional realm was the Swiss mathematician Ludwig Schläfli (1814-1895). He was less than well understood at his time, but anyway, in his "Theorie der vielfachen Kontinuität" (written 1850-1852, printed only in 1901 [10]) he stated the following theorem, nowadays called the Euler-Poincaré relation:

Theorem. For every d-dimensional convex polytope one has

$$
f_{0}-f_{1}+\ldots+(-1)^{d-1} f_{d-1}=1-(-1)^{d} .
$$

The first full proof of this theorem was given by Poincaré, as a consequence of his development of homology theory. The elementary "Ansatz" of Schläfli was only completed by Bruggesser and Mani [1] in 1969. It uses the method of "shelling", an induction procedure adding facets to the boundary 1-by-1.

3. Euler's formula (2) and its $d$-dimensional generalization (4) are necessary conditions that the $f$-vector $\left(f_{0}, f_{1}, \ldots, f_{d-1}\right)$ of a convex polytope must fulfill. Are there other conditions? In other words, we are faced with the following general problem:

Problem. Describe (characterize, approximate, ...) for given $d \geq 2$ the set

$$
\mathcal{F}_{d}:=\left\{\mathbf{f}=\left(f_{0}, f_{1}, \ldots, f_{d-1}\right) \mid \mathbf{f} \text { is the } f \text {-vector of a convex d-polytope }\right\} .
$$

The case $d=2$ is trivial: $f_{0}=f_{1} \geq 3$ is necessary and sufficient.

In the case $d=3$ one has the inequalities

$$
f_{2} \leq 2 f_{0}-4, \quad f_{0} \leq 2 f_{2}-4
$$

They follow from (2) and the fact that each facet resp. each vertex incides with at least 3 edges. In the case of simplicial polytopes (e.g., the icosahedron) we have $f_{2}=2 f_{0}-4$ and for simple polytopes (e.g., the cube) we have $f_{0}=2 f_{2}-4$. In 1906 Steinitz noted [11] that $\mathcal{F}_{3}$ is fully described by (2) and the conditions (5):

Theorem. A vector $\left(f_{0}, f_{1}, f_{2}\right) \in \mathbb{Z}^{3}$ is the $f$-vector of a 3-polytope if and only if the following conditions are satisfied:

$$
f_{0}-f_{1}+f_{2}=2, \quad f_{2} \leq 2 f_{0}-4, \quad f_{0} \leq 2 f_{2}-4 .
$$

Our Fig. 4 shows a projection of $\mathcal{F}_{3}$ into the $\left(f_{0}, f_{2}\right)$-plane. Because of (2), no information is lost under this representation. It appears that simplicial and simple polytopes form the "outer boundary" of $\mathcal{F}_{3}$. In higher dimensions simplicial and simple polytopes are still "extremal", so their $f$-vectors lie on the boundary of the corresponding cone (see Fig. 7), but for $d>3$ other types of extremal polytopes resp. $f$-vectors make the picture more complicated and more challenging.

In 1922 Steinitz proved a much deeper theorem on 3-polytopes, and even to this day no simple proof of this theorem has been found. Here one considers not the $f$-vector, a 


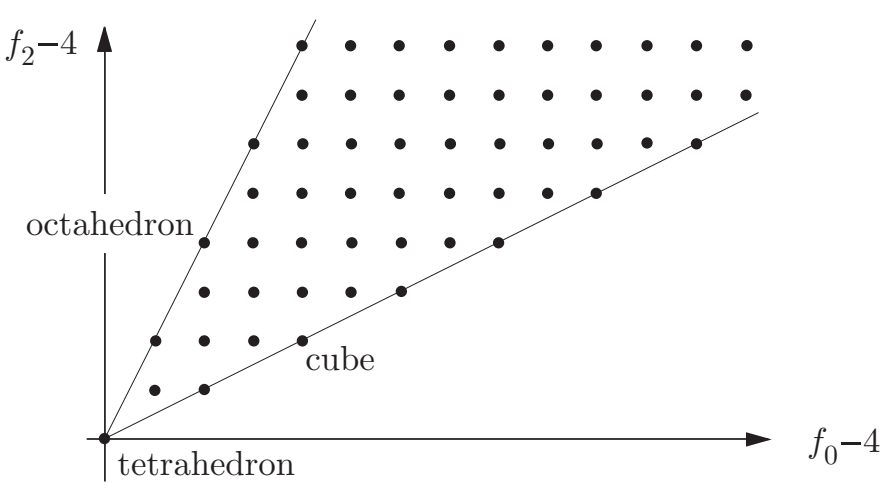

Figure 4

relatively coarse descriptor of a polytope, but the full information contained in the graph of its vertices and edges:

Problem. Characterize the graphs that can occur as 1-skeleton of a convex 3-polytope.

In his Encyclopaedia article [12] Steinitz gave the following definitive answer to this problem:

Theorem. A graph $\Gamma$ is the 1-skeleton of a 3-polytope if and only if it is simple, planar and 3-connected.

A graph is called 3-connected if any two of its vertices can be joined by three edge-paths which are pairwise disjoint, apart from the endpoints. (Check this out for a dodecahedron!)

4. We return to the problem of determining $\mathcal{F}_{d}$ in general. In $1971 \mathrm{McMullen}$, combining all information available at the time, formulated the so-called $g$-conjecture. This bold conjecture described in full the possible $f$-vectors of simplicial $d$-polytopes. McMullen's conjecture was proven in 1980. The proof (as in Steinitz' theorem above) involves two parts: For the necessity part, proven by Stanley, the "hard Lefschetz theorem for toric varieties with $V$-singularities" is put into action; for the sufficiency part, due to Billera $\&$ Lee, one has to construct an actual $d$-polytope for each $f$-vector that is theoretically admissible. Both parts of the proof are magnificent achievements!

We omit the details (!), but in order to convey the flavour of things we state what the $g$-theorem has to say in the case $d=4$ :

Theorem. The vector $\mathbf{f}=\left(f_{0}, f_{1}, f_{2}, f_{3}\right)$ is the $f$-vector of a simplicial convex 4 -polytope if and only if $\mathbf{f}$ can be represented in the following way:

$$
\mathbf{f}=\left(g_{0}, g_{1}, g_{2}\right)\left(\begin{array}{cccc}
5 & 10 & 10 & 5 \\
1 & 4 & 6 & 3 \\
0 & 1 & 2 & 1
\end{array}\right)
$$


for numbers $g_{i} \geq 0$ satisfying $g_{0}=1, g_{2} \leq\left(\begin{array}{c}g_{1}+1 \\ 2\end{array}\right)$.

To put it differently: The vector $\mathbf{f}$ has to be a certain non-negative linear combination of the rows in the above matrix $M$.
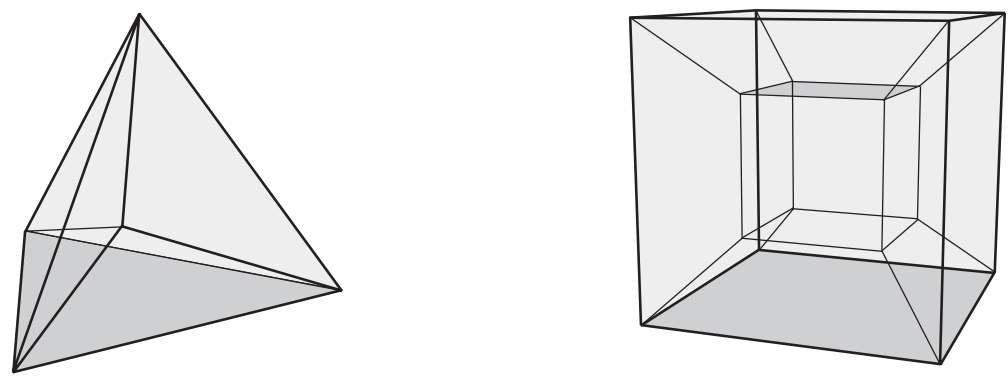

Figure 5

5. The above theorem describes the $f$-vectors of simplicial (and, by duality, of simple) 4-polytopes. As indicated before, these lay in a way on the "boundary" of $\mathcal{F}_{4}$. In the remaining part of this overview we now shall report about ongoing work concerning the $f$-vectors of arbitrary 4-dimensional polytopes, so we are looking for "the rest of the boundary" and thus try to capture the "bulk" of $\mathcal{F}_{4}$.

As Kalai [7] has noted, "it seems that overall, we are short of examples. The methods for coming up with useful examples (or counterexamples for commonly believed conjectures) are even less clear than the methods of proving." So let us start with a few examples of 4-polytopes. The figures, of a type known as "Schlegel diagrams", will not be as intuitive as one is accustomed to from 3 -space. The $f$-vectors $\left(f_{0}, \ldots, f_{3}\right)$ of the 4-simplex (Fig. 5, left) and the 4-cube (Fig. 5, right) are easily seen to be $(5,10,10,5)$ (compare the first row of the matrix $M !)$ and $(16,32,24,8)$, respectively. Then there is the "triangle $\times$ triangle" (Fig. 6, left), with $f$-vector $(9,18,15,6)$, and its generalization, the " $n$-gon $\times n$-gon", with $f$-vector $\left(n^{2}, 2 n^{2}, n^{2}+2 n, 2 n\right)$. In all dimensions there is the analogon of the octahedron, called cross polytope and defined by $X_{d}:=\left\{\mathbf{x} \in \mathbb{R}^{d}|| x_{1}|+\ldots+| x_{d} \mid \leq 1\right\}$. The $f$-vector of $X_{4}$ (Fig. 6, right) can, with some power of imagination, be read off as $(8,24,32,16)$; by duality, this is the "reverse" of the $f$-vector of the 4-cube.

A rich set of examples is provided by the so-called cyclic polytopes. These are obtained in the following way: Choose $n$ points $\mathbf{x}_{i}$ on the curve $t \mapsto\left(t, t^{2}, t^{3}, t^{4}\right)$ (or on a similar curve of degree 4 ) and let $P$ be the convex hull of the $\mathbf{x}_{i}$. The $\mathbf{x}_{i}$ will automatically be in convex position (consider the projection to the first two coordinates), and also in general position (this can be seen from Vandermonde determinants). Thus $P$ has $n$ vertices, and it is simplicial. The remarkable observation is that any two vertices are connected by an edge, so there are $\left(\begin{array}{l}n \\ 2\end{array}\right)$ edges. From this, one can derive the $f$-vector of $P$ to be

$$
\left(n, \frac{n^{2}-n}{2}, n^{3}-3 n, \frac{n^{2}-3 n}{2}\right)
$$



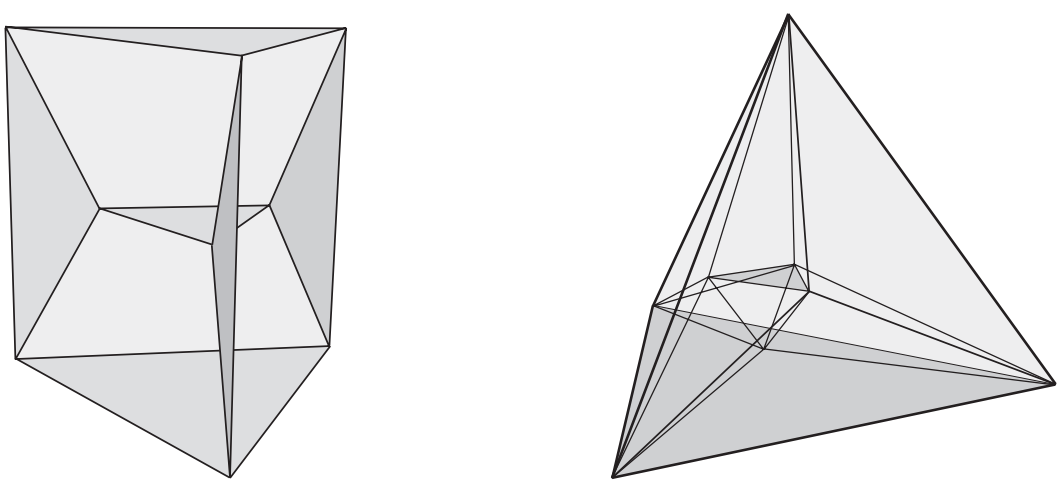

Figure 6

We could also mention the stacked 4-polytopes, constructed as follows: Start with a 4simplex $\Delta_{4}=: P_{0}$, then recursively add pyramids over facets. This leads to a sequence $\left(P_{n}\right)_{n \geq 0}$ of polytopes with $f$-vectors $(5+3 n, 10+6 n, 10+4 n, 5+n)$.

What to make of all of this? On the one hand we know necessary conditions: Apart from non-linear ones such as $f_{0} \leq \frac{1}{2} f_{3}\left(f_{3}-3\right)$, proven by Grünbaum [5], there are four linear conditions, easy to derive, namely $f_{1} \geq 2 f_{0}, f_{2} \geq 2 f_{3}, f_{0} \geq 5$, and $f_{3} \geq 5$. All of these are tight for the $f$-vector $\mathbf{f}\left(\Delta_{4}\right)=(5,10,10,5)$ of the simplex, so they describe a cone with this apex. On the other hand, all examples exhibited so far have $f$-vectors in a smaller cone with the same apex, spanned by the $f$-vectors of simplicial 4-polytopes (as given by the $g$-theorem for $d=4$ ) together with the $f$-vectors of simple 4-polytopes (which are just the same vectors, "reversed").

6. Because of the Euler-Poincaré relation (4) there are only three degrees of freedom in the vectors $\mathbf{f} \in \mathcal{F}_{4}$. If we focus on the linear conditions that are tight at the $f$-vector of the simplex, i.e., on the cone with apex $\mathbf{f}\left(\Delta_{4}\right)$ spanned by the $f$-vectors of 4-polytopes, then there is one more direction in our four-dimensional "moduli-space" $\mathcal{F}_{4}$ that can be factored out. One is lead to introduce the two "homogeneous" quantities

$$
\phi_{1}:=\frac{f_{1}-10}{f_{0}+f_{3}-10}, \quad \phi_{2}:=\frac{f_{2}-10}{f_{0}+f_{3}-10}
$$

which encode the size of the "middle coordinates" $f_{1}$ and $f_{2}$ of $\mathbf{f}$, compared to $f_{0}$ and $f_{3}$. The latter can be controlled to some extent at the moment the polytope is defined either as convex hull of finitely many points or as intersection of half-spaces. Fig. 7 shows the domain of admissible $\left(\phi_{1}, \phi_{2}\right)$-values, taking all so far established facts into account.

We now define the fatness $\Phi$ of a 4-polytope by

$$
\Phi:=\phi_{1}+\phi_{2}=\frac{f_{1}+f_{2}-20}{f_{0}+f_{3}-10} .
$$

The fatness of a polytope is large when it has many edges and 2-faces compared to the number of its vertices and facets. Looking at some of the examples above we obtain the following values: 


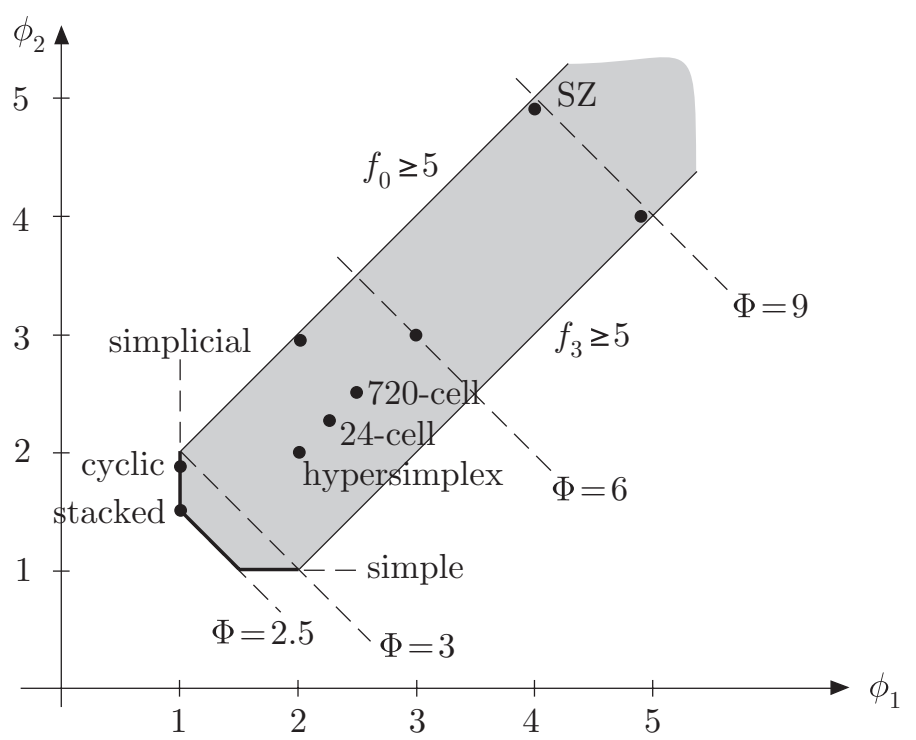

Figure 7

The 4-cube as well as its dual $X_{4}$ have fatness $\Phi=\frac{36}{14} \doteq 2.57$. The cyclic polytope with $n=8$ vertices has fatness $\Phi=\frac{48}{18} \doteq 2.67$, while all stacked polytopes have the same fatness $\frac{5}{2}$. It can easily be shown that all simplicial or simple 4-polytopes have fatness $<3$, which is best possible for these polytopes. But there are polytopes with fatness larger than that: The so-called "hypersimplex" with 30 vertices has fatness 4 and the famous 24-cell, invented by Schläfli, even fatness $\frac{172}{38} \doteq 4.526$. As a matter of fact, in the last few years a race for fatter and fatter polytopes has ensued.

In the light of these examples one hopes for general theorems. A lower bound is provided by the following theorem by Kalai, 1987 [6]:

Theorem. All convex 4-polytopes $P$ have fatness $\Phi(P) \geq \frac{5}{2}$.

For results in the other direction explicit constructions are needed. In this regard one has the following theorem by Sanyal and the first author, 2004 [13], 2007 [9]:

Theorem. For $r \geq 2$ and even $n \geq 4$ there is a realization $\widetilde{C}_{n}^{r} \subset \mathbb{R}^{2 r}$ of the $r$-fold product of $n$-gons $C_{n} \subset \overline{\mathbb{R}}^{2}$, such that all vertices, all edges, and all $n$-gon 2-faces of $\widetilde{C}_{n}^{r}$ survive an appropriate projection $\pi: \mathbb{R}^{2 r} \rightarrow \mathbb{R}^{4}$.

As a corollary one obtains the fattest known 4-polytopes so far:

Corollary. There are convex 4-polytopes $\pi\left(\widetilde{C}_{n}^{r}\right)$ with $f$-vector approximately proportional to $(0,4,5,1)$ and fatness arbitrarily close to 9 .

Is 9 the upper limit? We don't know. Kalai was right: We are short of examples! 


\section{References}

[1] Bruggesser, H.; Mani, P.: Shellable decompositions of cells and spheres. Math. Scand. 29 (1971), 197205

[2] Descartes, R.: Exercices pour les éléments des solides. Ed. and transl. by Pierre Costabel, Presses Universitaires de France, 1987.

[3] Eppstein, D.: Nineteen proofs of Euler's formula $V-E+F=2$. The geometry junkyard, http: // www. ics.uci.edu/ eppstein/junkyard/euler/

[4] Federico, P.J.: Descartes on polyhedra. A study of the 'De Solidorum Elementis'. Springer, 1982.

[5] Grünbaum, B: Convex polytopes. Vol. 221 of Graduate Texts in Mathematics, Springer, 2003. (Original ed.: Interscience, 1967. )

[6] Kalai, G.: Rigidity and the lower bound theorem, I. Invent. Math. 88 (1987), 125-151.

[7] Kalai, G.: Combinatorics with a geometric flavor. GAFA 2000 (Tel Aviv 1999). Geom. Funct. Anal., Special volume, part II (2000), 742-791.

[8] Koch, H.: Der Briefwechsel von Leonhard Euler und Christian Goldbach. Elem. Math. 62 (2007), 155166.

[9] Sanyal, R.; Ziegler, G.M.: Construction and analysis of projected deformed products. Preprint, October 2007.

[10] Schläfli, L.: Theorie der vielfachen Kontinuität. Zürcher und Furrer, Zürich, 1901. (Reprinted in: Ludwig Schläfl, 1814-1895, Gesammelte Mathematische Abhandlungen, Vol. I, 167-387. Birkhäuser, 1950.)

[11] Steinitz, E.: Über die Eulerschen Polyederrelationen. Archiv für Mathematik und Physik 11 (1906), 86-88.

[12] Steinitz, E.: Polyeder und Raumeinteilungen. Encyclopädie der mathematischen Wissenschaften. Band 3 (Geometrie), Teil 3AB12,1-139. Teubner, 1922.

[13] Ziegler, G.M.: Projected products of polygons. Electron. Res. Announc. Amer. Math. Soc. 10 (2004), 122-134, http: //arXiv.org/abs/math/ 0407042.

Günter M. Ziegler

Inst. Mathematics, MA 6-2

Techn. Universität Berlin

D-10623 Berlin, Germany

e-mail: ziegler@math.tu-berlin.de

Christian Blatter

Albertus-Walder-Weg 16

CH-8606 Greifensee, Switzerland

e-mail: christian.blatter@math.ethz.ch 